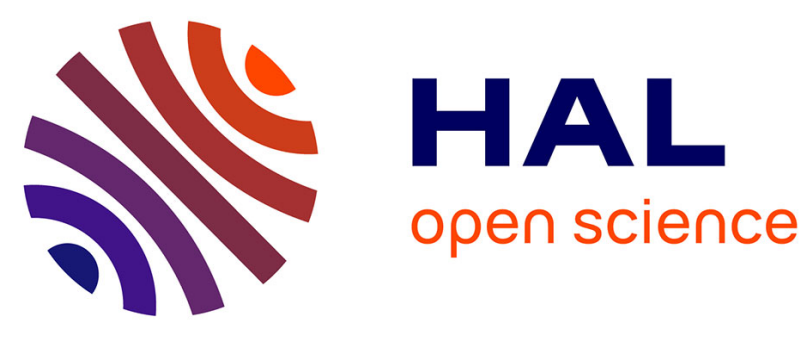

\title{
Feasibility and individual rationality in two-person Bayesian games
}

Francoise Forges, Ulrich Horst, Antoine Salomon

\section{To cite this version:}

Francoise Forges, Ulrich Horst, Antoine Salomon. Feasibility and individual rationality in two-person Bayesian games. 2014. hal-01094061

\section{HAL Id: hal-01094061 https://hal.science/hal-01094061}

Preprint submitted on 11 Dec 2014

HAL is a multi-disciplinary open access archive for the deposit and dissemination of scientific research documents, whether they are published or not. The documents may come from teaching and research institutions in France or abroad, or from public or private research centers.
L'archive ouverte pluridisciplinaire HAL, est destinée au dépôt et à la diffusion de documents scientifiques de niveau recherche, publiés ou non, émanant des établissements d'enseignement et de recherche français ou étrangers, des laboratoires publics ou privés. 


\title{
Feasibility and individual rationality in two-person Bayesian games*
}

\author{
Françoise Forges Ulrich Horst $^{\ddagger}$ and Antoine Salomon ${ }^{\S}$
}

November 25, 2014

\begin{abstract}
We define feasible, posterior individually rational solutions for twoperson Bayesian games with a single informed player. Such a solution can be achieved by direct signalling from the informed player and requires approval of both players after the signal has been sent. Without further assumptions on the Bayesian game, a solution does not necessarily exist. We show that, if the uninformed player has a "uniform punishment strategy" against the informed one, the existence of a solution follows from the existence of Nash equilibrium in infinitely repeated games with lack of information on one side. We consider the extension of the result when both players have private information.
\end{abstract}

${ }^{*}$ We are grateful to Gorkem Celik, Marie Laclau, Antoine Loeper, Jérôme Renault, Rann Smorodinsky, Bernhard von Stengel, Roland Strausz and seminar participants at Vanderbilt University for useful comments. F. Forges acknowledges support from Institut Universitaire de France. U. Horst acknowledges financial support through SFB 649 Economic Risk.

${ }^{\dagger}$ PSL, Université Paris-Dauphine, CEREMADE and LEDa. E-mail: francoise.forges@gmail.com

†Department of Mathematics, Humboldt University Berlin. E-mail: horst@math.huberlin.de

§PSL, Université Paris-Dauphine, LEDa. E-mail: antoine.salomon@dauphine.fr 


\section{Introduction}

The classical folk theorem states that the set of Nash equilibrium payoffs of an undiscounted infinitely repeated game with complete information coincides with the set of feasible and individually rational payoffs of the one-shot game. As emphasized in Myerson (1991), Kalai et al. (2010) and Tennenholtz (2004), the latter set describes meaningful solutions for one-shot games with commitment, namely, cooperative solutions of the one-shot game. In this paper, we investigate the possible relationship between noncooperative solutions of the infinitely repeated game and cooperative solutions of the one-shot game when information is incomplete.

We mostly consider a two-person Bayesian game with a single informed player. In such a model, feasibility and individual rationality can take various forms. We introduce feasible, posterior individually rational solutions, which can be achieved by direct signalling from the informed player (i.e., by cheap talk, without the help of a mediator) and are approved by both players after the informed player has sent his signal (i.e., given the posterior belief of the uninformed player). This set is smaller than the set of mediated, incentive compatible, interim individually rational solutions of Myerson (1991), which is always nonempty.

Without further assumptions on the Bayesian game, we show that a feasible, posterior individually rational solution (which will just be referred to as a "solution" when there is no confusion) does not necessarily exist. If the informed player has no decision to make, the problem reduces to a senderreceiver game and there always exists a nonrevealing solution. If the uninformed player knows his own payoff (private values), then there always exists a completely revealing solution. We propose another, more interesting, assumption that guarantees the existence of a solution: the uninformed player has a "uniform punishment strategy" (UPS) against the informed one, namely, the uninformed player has a strategy that keeps the informed player at his type-dependent individually rational level, whatever his type. In this case, we show on an example that there may be only partially revealing solutions.

The existence of a feasible, posterior individually rational solution under UPS can be deduced from the existence of a Nash equilibrium in every twoperson undiscounted infinitely repeated game with lack of information on one side. This class of games was introduced by Aumann, Maschler and Stearns $(1968,1995)$ who identified a particular kind of Nash equilibrium, 
the joint plan equilibrium, in which there is a single phase of information transmission from the informed player to the uninformed one. Simon et al. (1995) established the existence of a joint plan equilibrium by making use of sophisticated mathematical tools. Sorin (1983) first proved the result under the assumption that the informed player has two possible types. In the case of private values, the existence of a completely revealing Nash equilibrium is straightforward, as shown by Shalev (1994) (see Forges (1992), Hart (1985), Renault (2000), Simon (2002) and Simon et al. (2008) for further results on nonzero sum repeated games with incomplete information).

We can interpret our result as an extension of the folk theorem to twoperson games with a single informed player: under UPS, a set of noncooperative solutions of the infinitely repeated game (i.e., the joint plan equilibrium payoffs) coincides with a set of feasible, individually rational solutions of the one-shot game (feasibility being understood without a mediator and individual rationality being understood a posteriori). However, when the UPS assumption does not hold, such solutions may not exist in the one-shot game, while joint plan equilibria always exist in the infinitely repeated one.

There is a large literature on cooperative solutions to Bayesian games and we will not try to survey it here. The main differences between the present paper and others is that we consider situations in which

(i) the informed player(s) can only transmit information by cheap talk

(ii) both players can decline participation at the very last stage of the contracting process, i.e., after information has been transmitted.

In Myerson (1991)'s approach, individual rationality is formulated at the interim stage, i.e., before mediated information transmission, but further properties are imposed, e.g., appropriate forms of Pareto efficiency. Peters and Celik (2011) and Peters and Szentes (2012) extend Bayesian games by means of specific (noncooperative) negotiation schemes, in which the players simultaneously exchange information and partially commit; posterior individual rationality need not hold. A. Kalai and E. Kalai (2013) propose the cooperative "coco" solution, which, under suitable assumptions, can be implemented by direct information transmission, with participation constraints at the ex ante or interim stage.

The paper is organized as follows: in section 2, we define feasible, posterior individually rational solutions; in section 3 , we address the question of the 
existence of posterior individually rational solutions in two-person Bayesian games and we show that UPS guarantees it by making the connection with infinitely repeated games; in section 4 , we propose further elements of comparison between the one-shot game and the repeated game and we consider the possible extension of our results to two-person Bayesian games in which both players have private information.

\section{Individually rational solutions in Bayesian games with a single informed player}

\subsection{Basic framework and feasible solutions}

Our basic framework is a two-person Bayesian game in which only player 1 has private (nonverifiable) information. Let $K$ be a finite set. A type $k$ is chosen in $K$ according to $p \in \Delta(K)$ at a virtual initial stage of the game and only player 1 is informed of $k{ }^{1}$ Unless specified otherwise, we assume that $p^{k}>0$ for every $k \in K$. Player 1 and player 2 choose simultaneously an action in finite sets $A_{1}$ and $A_{2}$ respectively. If the pair of actions $a \in A=A_{1} \times A_{2}$ is chosen, they get the respective payoffs $U^{k}(a)$ and $V^{k}(a)$. We refer to this game as $B(p)$.

We are going to define a solution for $B(p)$, which will be cooperative in the sense that it specifies a binding agreement between the players. ${ }^{2}$ A solution is described by a finite set $S$ of signals, a signalling strategy $\mu: K \rightarrow \Delta(S)$ for the informed player and a joint decision function $\chi: S \rightarrow \Delta(A)$, which associates a correlated strategy $\chi(s)=x_{s} \in \Delta(A)$ with every signal $s \in S$. The interpretation is that after player 1 has sent the signal $s$, actions are chosen in $A$ on behalf of the players, according to the lottery $x_{s}$.

The description of a solution in terms of mappings $\mu$ and $\chi$ incorporates basic feasibility conditions. Meaningful feasibility also requires that the informed player's signalling strategy be incentive compatible. For every $k \in K$, let $\mu(k)=\mu(\cdot \mid k)$ be the probability distribution over $S$ associated with the signalling strategy $\mu$. Together with the prior probability $p$ over $K, \mu$ induces the probability distribution $(p \times \mu)(s)=\sum_{k \in K} p^{k} \mu(s \mid k)$ over $K \times S$. Let

\footnotetext{
${ }^{1}$ For any finite set $E, \Delta(E)$ denotes the set of probability distributions over $E$.

${ }^{2}$ The word "solution" may look too strong and is just used for convenience. As will become clear below, we do not make a list of all properties that a solution should satisfy but concentrate on two of them.
} 
$p_{s}=\left(p_{s}^{k}\right)_{k \in K}$ be the posterior probability induced by $p$ and $\mu$ over $K$; $p_{s}$ is well-defined as soon as $(p \times \mu)(s)>0$, i.e., given our assumption that $p^{k}>0$ for every $k \in K$, as soon as $\exists k: \mu(s \mid k)>0$. By restricting $S$, we can assume that $(p \times \mu)(s)>0$ for every $s \in S$. We still use the notation $U^{k}$ and $V^{k}$ to denote the extension of the payoff functions to $\Delta(A)$.

The signalling strategy $\mu: K \rightarrow S$ is incentive compatible (given $\chi$ ) iff

i) $\forall k \in K, \forall s, s^{\prime} \in S$ such that $\mu(s \mid k)>0$ and $\mu\left(s^{\prime} \mid k\right)>0$,

$$
U^{k}\left(x_{s}\right)=U^{k}\left(x_{s^{\prime}}\right)
$$

ii) $\forall k \in K, \forall s, s^{\prime} \in S$ such that $\mu(s \mid k)>0$ and $\mu\left(s^{\prime} \mid k\right)=0$,

$$
U^{k}\left(x_{s}\right) \geq U^{k}\left(x_{s^{\prime}}\right)
$$

Conditions i) and ii) take into account that player 1 sends a random signal by himself, without the help of a mediator; hence he randomizes over signals $s, s^{\prime} \in S$ if and only if he is indifferent between $s$ and $s^{\prime}$.

Incentive compatibility implies in particular that for all types $k$, all signals $s$ sent by type $k$ (i.e., such that $\mu(s \mid k)>0$ ) lead to the same payoff. Let us set

$$
u^{k} \equiv U^{k}\left(x_{s}\right) \forall s \in S \text { such that } \mu(s \mid k)>0 .
$$

The payoff $u^{k}$ is the final interim expected payoff of player 1 when his type is $k$ and it coincides with his posterior payoff, given $s$, for every relevant $s$.

\subsection{Individual rationality}

We will require that, in addition to being feasible, a solution be acceptable, namely, individually rational, for both parties. This means that each player should get at least what he can guarantee to himself by playing the original one-shot Bayesian game without cooperating. This motivates the following definitions of individual rationality. For player 1 , the vector payoff matters so that the definition does not depend on the underlying probability distribution over $K$.

Definition 1 A payoff vector $u=\left(u^{k}\right)_{k \in K}$ is (interim) individually rational for player 1 in $B(\cdot)$ if player 2 has a strategy in $B(\cdot)$ that prevents player 1 from getting more than $u^{k}$ when his type is $k$, namely

$$
\exists \tau \in \Delta\left(A_{2}\right) \forall k \in K \forall \sigma \in \Delta\left(A_{1}\right): U^{k}(\sigma, \tau) \leq u^{k}
$$


This definition reduces to

$$
u \geq \min _{\tau \in \Delta\left(A_{2}\right)} \max _{\sigma \in \Delta\left(A_{1}\right)} U(\sigma, \tau)
$$

in the case of complete information, namely, when $|K|=1$.

In a similar way,

Definition 2 Let $q \in \Delta(K)$. A payoff $\bar{v}$ is individually rational for player 2 in $B(q)$ if player 1 has a strategy in $B(q)$ that prevents player 2 from getting more than $\bar{v}$, namely

$$
\exists \widetilde{\sigma}=\left(\widetilde{\sigma}^{k}\right)_{k \in K} \in \Delta\left(A_{1}\right)^{K} \forall \tau \in \Delta\left(A_{2}\right): \sum_{k} q^{k} V^{k}\left(\widetilde{\sigma}^{k}, \tau\right) \leq \bar{v}
$$

Individual rationality can be defined in an equivalent but more tractable way. ${ }^{3}$ Let, for every $q \in \Delta(K)$,

$$
\begin{aligned}
f_{1}(q) & =\max _{\tilde{\sigma} \in \Delta\left(A_{1}\right)^{K}} \min _{\tau \in \Delta\left(A_{2}\right)} \sum_{k} q^{k} U^{k}\left(\widetilde{\sigma}^{k}, \tau\right)=\min _{\tau \in \Delta\left(A_{2}\right)} \sum_{k} q^{k} \max _{\sigma \in \Delta\left(A_{1}\right)} U^{k}(\sigma, \tau) \\
& =\min _{\tau \in \Delta\left(A_{2}\right)} \sum_{k} q^{k} \max _{a_{1} \in A_{1}} U^{k}\left(a_{1}, \tau\right)
\end{aligned}
$$

$f_{1}(q)$ is the value of the one-shot zero-sum Bayesian game $\Gamma_{1}(q)$ in which player 1's type $k$ is chosen according to $q$ and payoffs are defined by $U^{k}$ for player 1 and $-U^{k}$ for player $2 .{ }^{4}$ Of course, player 1 can make use of his private information in $\Gamma_{1}(q)$. As a minimum of linear functions (of $q$ ), $f_{1}$ is concave over $\Delta(K)$.

Lemma 3 A payoff vector $u=\left(u^{k}\right)_{k \in K}$ is (interim) individually rational for player 1 in $B(\cdot)$ if and only if

$$
\forall q \in \Delta(K) \quad q \cdot u \geq f_{1}(q)
$$

where $f_{1}$ defined by (4).

\footnotetext{
${ }^{3}$ The properties detailed below are well identified in Sorin (2002).

${ }^{4}$ The index 1 in $f_{1}(\cdot)$ is for "one-shot".
} 
Proof: We first observe that (2) is equivalent to

$$
\exists \tau \in \Delta\left(A_{2}\right) \forall q \in \Delta(K): \sum_{k} q^{k} \varphi^{k}(\tau) \leq \sum_{k} q^{k} u^{k}
$$

where $\varphi^{k}(\tau)$ is defined as $\varphi^{k}(\tau)=\max _{\sigma \in \Delta\left(A_{1}\right)} U^{k}(\sigma, \tau), k \in K$.

On the other hand, from the definition of $f_{1}$,

$$
f_{1}(q)=\min _{\tau \in \Delta\left(A_{2}\right)} \sum_{k} q^{k} \varphi^{k}(\tau)
$$

so that (5) is equivalent to

$$
\forall q \in \Delta(K) \exists \tau \in \Delta\left(A_{2}\right): \sum_{k} q^{k} \varphi^{k}(\tau) \leq \sum_{k} q^{k} u^{k}
$$

Using that $\varphi^{k}(\cdot)$ is convex for every $k$, one shows that $(6) \Leftrightarrow(7)$, either by using a separation argument (see Sorin (2002), proposition 2.7) or by using Sion's minmax theorem on an auxiliary game:

$$
\begin{aligned}
& (7) \Leftrightarrow \max _{q} \min _{\tau} \sum_{k}\left[q^{k}\left(\varphi^{k}(\tau)-u^{k}\right)\right] \leq 0 \\
& (6) \Leftrightarrow \min _{\tau} \max _{q} \sum_{k}\left[q^{k}\left(\varphi^{k}(\tau)-u^{k}\right)\right] \leq 0
\end{aligned}
$$

According to the new formulation (5), a vector payoff $u$ is individually rational for player 1 if the hyperplane defined by $u$, namely $q \cdot u$, is above $f_{1}$ over the whole simplex $\Delta(K)$. This property is much stronger than individual rationality at every type. Let us denote as $\delta_{k}$ the $k^{\text {th }}$ extreme point of $\Delta(K)$ (i.e., $\delta_{k}^{k}=1, \delta_{k}^{\ell}=0$ for $\ell \neq k$ ) and let us set $\underline{u}^{k}=f_{1}\left(\delta_{k}\right)$, namely,

$$
\underline{u}^{k}=\min _{\tau \in \Delta\left(A_{2}\right)} \max _{\sigma \in \Delta\left(A_{1}\right)} U^{k}(\sigma, \tau)=\max _{\sigma \in \Delta\left(A_{1}\right)} \min _{\tau \in \Delta\left(A_{2}\right)} U^{k}(\sigma, \tau)
$$

A payoff $u^{k}$ is individually rational for every type $k$ if $u^{k} \geq \underline{u}^{k}$ (i.e., assuming complete information of type $k$ ). If $u$ is individually rational for player 1 , then for every $k \in K, u^{k} \geq \underline{u}^{k}$. But the reverse is not true: the hyperplane defined by the vector $\underline{u}=\left(\underline{u}^{k}\right)_{k \in K}$ might not be above $f_{1}$, although $\underline{u}^{k} \geq f_{1}\left(\delta_{k}\right)$ for every $k$ (see the example in section 3.1 below).

Let us proceed in a similar way for player 2. Let $g_{1}(q)$ be the value of the one-shot ${ }^{5}$ zero-sum Bayesian game $\Gamma_{2}(q)$ in which player 1's type $k$ is

\footnotetext{
${ }^{5}$ As for the informed player, the index 1 in $g_{1}(\cdot)$ is for "one-shot".
} 
chosen according to $q$, player 1 can make use of his information and payoffs are defined by $-V^{k}$ for player 1 and $V^{k}$ for player 2, namely, for every $q \in \Delta(K)$,

$$
\begin{aligned}
g_{1}(q) & =\min _{\widetilde{\sigma} \in \Delta\left(A_{1}\right)^{K}} \max _{\tau \in \Delta\left(A_{2}\right)} \sum_{k} q^{k} V^{k}\left(\widetilde{\sigma}^{k}, \tau\right)=\max _{\tau \in \Delta\left(A_{2}\right)} \sum_{k} q^{k} \min _{\sigma \in \Delta\left(A_{1}\right)} V^{k}(\sigma, \tau) \\
& =\max _{\tau \in \Delta\left(A_{2}\right)} \sum_{k} q^{k} \min _{a_{1} \in A_{1}} V^{k}\left(a_{1}, \tau\right)
\end{aligned}
$$

As a maximum of linear functions (of $q$ ), $g_{1}$ is convex over $\Delta(K)$. The next result is immediate:

Lemma 4 A payoff $\bar{v}$ is individually rational for player 2 in $B(q)$ iff $\bar{v} \geq$ $g_{1}(q)$, where $g_{1}$ is defined by (9).

\subsection{Feasible, posterior individually rational solutions}

We are now ready to define a solution for $B(p)$.

Definition 5 Let $B(p)$ be a two-person Bayesian game with a single informed player. A (cooperative) solution $(S, \mu, \chi)$ for $B(p)$ consists of a set of signals $S$, a signalling strategy $\mu: K \rightarrow \Delta(S)$ for the informed player and of a joint decision function $\chi: S \rightarrow \Delta(A)$ such that $\mu$ is incentive compatible given $\chi$, player 1's interim expected payoff is individually rational and for every signal $s \in S$, player 2's payoff from $\chi(s)=x_{s}$ is individually rational in $B\left(p_{s}\right)$, where $p_{s}$ denotes player 2's posterior computed from $p$ and $\mu$. $\mathcal{C}[B(p)] \subseteq \mathbb{R}^{K} \times \mathbb{R}$ denotes the set of all interim expected payoffs that can be achieved by a solution of $B(p)$.

Henceforth, we refer to a triple $(S, \mu, \chi)$ satisfying definition 5 as a "feasible, posterior individually rational solution" or simply as a "solution". For player 2 , individual rationality is indeed readily stated in terms of the posterior probability distribution given every signal. This participation condition is appropriate if player 2 does not fully commit himself before observing the type dependent signal of player 1 . For the latter player, standard interim individually rational is required but, given the strong incentive compatibility conditions, the interim expected payoff $u^{k}$ and the posterior payoff $U^{k}\left(x_{s}\right)$ coincide as soon as the latter payoff is relevant (namely, as soon as $\mu(s \mid k)>0$ ). 
The next lemma states that every solution can be implemented as a Nash equilibrium of a noncooperative game, in which player 1 sends a signal and then both players agree on a joint decision, conditionally on the signal. This implementation procedure offers a justification for the previous formulation of feasibility (involving incentive compatibility) and individual rationality.

Given the basic game $B(p)$, a set of signals $S$ and a joint decision function $\chi: S \rightarrow \Delta(A)$, let $G(p, S, \chi)$ be the noncooperative game described as follows:

- Player 1's type $k$ is chosen in $K$ according to $p$, only player 1 is informed of $k$.

- Player 1 sends a signal $s \in S$ to player 2 .

- The correlated strategy $\chi(s)=x_{s} \in \Delta(A)$ is proposed to both players.

- The players simultaneously accept or reject the correlated strategy $x_{s}$.

- If both players accept $x_{s}$, player 1 gets $U^{k}\left(x_{s}\right)$ and player 2 gets $V^{k}\left(x_{s}\right)$.

- If at least one of the players rejects $x_{s}$, player 1 chooses $a_{1}$, player 2 chooses $a_{2}$, player 1 gets $U^{k}\left(a_{1}, a_{2}\right)$ and player 2 gets $V^{k}\left(a_{1}, a_{2}\right)$.

Lemma 6 Let $B(p)$ be a two-person Bayesian game with a single informed player. $(S, \mu, \chi)$ defines a solution for $B(p)$ if and only if $G(p, S, \chi)$ has an equilibrium in which player 1 sends his signal according to $\mu$ and both players always accept the correlated strategy that is proposed to them.

Proof: To complete the description of strategies in $G(p, S, \chi)$ in which player 1's signalling strategy is $\mu$ and both players always accept the correlated strategy, let player 1's decision strategy be $\sigma: K \times S \rightarrow \Delta\left(A_{1}\right)$ in case of rejection and let player 2's decision strategy $\tau: S \rightarrow \Delta\left(A_{2}\right)$ in case of rejection.

Let us set

$$
\begin{aligned}
y_{s}^{k} & =\max _{a_{1}} U^{k}\left(a_{1}, \tau(s)\right) \\
b_{s} & =\max _{a_{2}} \sum_{k} p_{s}^{k} V^{k}\left(\sigma^{k}(s), a_{2}\right)
\end{aligned}
$$


Player 1's equilibrium conditions are, for every type $k$ :

$\forall s \in S$ such that $\mu(s \mid k)>0, \forall s^{\prime} \in S$ (even such that $\left.\mu\left(s^{\prime} \mid k\right)=0\right)$ : $U^{k}\left(x_{s}\right) \geq U^{k}\left(x_{s^{\prime}}\right)$ and $U^{k}\left(x_{s}\right) \geq y_{s^{\prime}}^{k}$.

Indeed, player 1 can consider any signal $s^{\prime}$, and, having sent $s^{\prime}$, can accept or reject $x_{s^{\prime}}$; in the latter case, player 1 cannot do better than best replying to $\tau\left(s^{\prime}\right)$. Player 1's equilibrium conditions are thus equivalent to incentive compatibility (as defined by i) and ii)) together with

$$
u^{k} \geq y_{s}^{k} \quad \text { for every } k \in K, s \in S
$$

where $u=\left(u^{k}\right)_{k \in K}$ is defined by (1). Let us check that $u$ satisfies (10) if and only if $u$ is individually rational in the sense of definition 1 .

Assume that $u$ satisfies (10). For every $s, y_{s}=\left(y_{s}^{k}\right)_{s \in S}$ is individually rational in the sense of definition 1 (which is satisfied with $\tau=\tau(s)$ ). Hence, $u \geq y_{s}$ is also individually rational in the sense of definition 1 .

Conversely, if $u$ is individually rational in the sense of definition 1 , there is a strategy $\tau$ of player 2 for which this definition is satisfied. Let $\tau(s)=\tau$ for every $s$; then $y_{s}^{k}=y^{k}=\max _{a_{1}} U^{k}\left(a_{1}, \tau\right) \leq u^{k}$.

Let us come to player 2's equilibrium conditions. Given the signal $s$, he updates his probability distribution over $K$ to $\left(p_{s}^{k}\right)_{k \in K}$. By rejecting $x_{s}$ and best replying to $\sigma(s): K \rightarrow \Delta\left(A_{1}\right)$, he gets $b_{s}$. Hence player 2 accepts $x_{s}$ if and only if

$$
\sum_{k} p_{s}^{k} V^{k}\left(x_{s}\right) \geq b_{s}
$$

This is equivalent to $\sum_{k} p_{s}^{k} V^{k}\left(x_{s}\right)$ is individually rational for player 2 in $B\left(p_{s}\right)$, in the sense of definition 2 .

Even if every solution can be seen as a Nash equilibrium of some game, this equilibrium is a quite demanding one and we should not expect standard existence theorems to apply. We will turn to the existence issue in the next section. Before that, we recall a standard approach to feasibility and individual rationality, which is quite different from the previous one.

\subsection{Mediated interim individually rational solutions}

In the game $G(p, S, \chi)$, the players only rely on a device (namely, $\chi$ ) to perform the lottery over joint actions conditionally on the informed player's

\footnotetext{
${ }^{6}$ This just shows that player 2's decision strategy in case of rejection does not have to depend on the signal $s$.
} 
signal but do not use any device to communicate. In other words, player 1 has to send his signal by himself (by means of $\mu$ ). Moreover, player 1 can only make use of his private information in the final decision by sharing it with player 2 .

Myerson (1991, chapter 6, specially section 6.6) considers mediated solutions, in which player 1 is invited to report his information $k$ to a mediator, who directly chooses a joint decision in $\Delta(A)$ on behalf of the players. ${ }^{7}$ The mediator's strategy can thus be described as a device $\pi: K \rightarrow \Delta(A)$. Incentive compatibility just says that player 1 cannot benefit from lying to the mediator, namely

$$
U^{k}(\pi(k)) \geq U^{k}\left(\pi\left(k^{\prime}\right)\right) \quad \text { for every } k, k^{\prime} \in K
$$

At a mediated solution, both players accept the help of the mediator at the interim stage, namely, before the mediator implements the device $\pi$. Myerson (1991) thus requires that player 1's interim expected payoff $\left(U^{k}(\pi(k))\right)_{k \in K}$ be individually rational according to definition 1 (namely, satisfies (5)) and player 2's expected payoff be individually rational according to definition 2 .

Definition 7 Let $B(p)$ be a two-person Bayesian game with a single informed player. A mediated solution for $B(p)$ consists of a device $\pi: K \rightarrow$ $\Delta(A)$ such that $\pi$ is incentive compatible, player 1's interim expected payoff is individually rational and player 2 's expected payoff $\sum_{k} p^{k} V^{k}(\pi(k))$ is individually rational in $B(p) . \mathcal{M}[B(p)] \subseteq \mathbb{R}^{K} \times \mathbb{R}$ denotes the set of all interim expected payoffs that can be achieved by a mediated solution in $B(p)$.

$\mathcal{M}[B(p)]$ contains the set of Nash interim expected equilibrium payoffs of $B(p)$ and is thus not empty. ${ }^{8}$ By proceeding as in the revelation principle (see Myerson (1991)), one can show that $\mathcal{C}[B(p)] \subseteq \mathcal{M}[B(p)]$, i.e., payoffs to unmediated posterior individually rational solutions can be achieved with the help of a mediator. Forges (2013) shows that $\mathcal{M}[B(p)]$ can be interpreted as the set all Nash equilibrium payoffs that the players can achieve by making conditional commitments at the interim stage, a result that was established by Kalai et al. (2010) in the case of complete information.

\footnotetext{
${ }^{7}$ As soon as there is a mediator, the revelation principle applies and without loss of generality, player 1's possible signals are just his possible types.

${ }^{8}$ Nonemptiness of $\mathcal{M}[B(p)]$ can also be checked directly, since a mediated solution is defined by linear inequalities. Myerson starts by defining $\mathcal{M}[B(p)]$ but goes on by imposing further requirements on cooperative solutions.
} 


\section{Existence of feasible, posterior individually rational solutions}

\subsection{A counter-example}

Let us illustrate the properties defined above and let us show that without further assumptions on the Bayesian game, incentive compatibility (in the strong sense of section 2.1) and posterior individual rationality may contradict each other.

Example 1: Let $B(p)$ be defined by $K=\{1,2\}, A_{1}=\{T, B\}, A_{2}=\{L, R\}$ and the following payoff matrices:

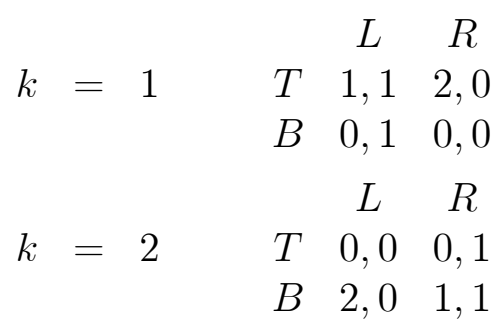

Let us first show that, even without taking account of player 2's payoffs, there cannot be any nonrevealing joint decision that is individually rational for player 1. Indeed, any distribution over $A=\{T, B\} \times\{L, R\}$ gives a sum of expected payoffs $u^{1}+u^{2} \leq 2$ to player 1 . If the original Bayesian game is played noncooperatively, player 2 plays $L$ with some probability $\alpha$. By playing $T$ when $k=1$ and $B$ when $k=2$, player 1 gets $\alpha+2(1-\alpha)$ when $k=1$ and $2 \alpha+(1-\alpha)$ when $k=2$ and the sum of his expected payoffs is 3 .

Similarly, if player 1 sends a signal $s$ that leads to an interior posterior probability distribution, by incentive compatibility, we must have $u^{k}=$ $U^{k}\left(x_{s}\right), k=1,2$, where $x_{s}$ is a distribution over $\{T, B\} \times\{L, R\}$, and again $u$ cannot be individually rational for player 1 .

The only way to possibly get an individually rational payoff vector for player 1 is thus a completely revealing solution. Such a solution is individually rational for player 2 iff $L$ has probability 1 when type 1 is reported, and $R$ has probability 1 when type 2 is reported. This can e.g. be seen from definition 2 and lemma 4: an expected payoff $\bar{v}$ is individually rational for player 2 in $B(q)$ iff $\bar{v} \geq \max \{q, 1-q\}$. This implies that player 1 gets at most 1 , whatever his type is. But $(1,1)$ is not an individually rational vector payoff for player 1 , since we have seen above that player 1 
could guarantee 3 as a sum of expected payoffs. Actually, according to (4), $f_{1}(q)=\min \{q+2(1-q), 2 q+(1-q)\}$; a vector payoff $\left(u^{1}, u^{2}\right)$ is thus individually rational for player 1 iff $u^{1}+u^{2} \geq 3$ but taking account of feasibility, this reduces to $u^{1}+u^{2}=3$.

Observe that, according to (8), $\underline{u}^{1}=\underline{u}^{2}=1$ in this example, which illustrates well that $u^{1} \geq 1$ and $u^{2} \geq 1$ is not enough for $\left(u^{1}, u^{2}\right)$ to be individually rational for player 1 . Playing $(T, L)$ if $k=1$ and $(B, R)$ if $k=2$ is incentive compatible and naïvely individually rational for player 1 . But player 1 will not accept such a contract if he has the possibility of just playing the original game by himself.

\subsection{Particular cases where a solution is easily shown to exist}

\subsubsection{Sender-receiver games}

Let us assume that the informed player has no decision to make in the basic Bayesian game $B(p)$, namely, that $A_{1}$ is a singleton (equivalently, the informed player has several actions but they have no impact on the payoffs). In this case, we can set $A_{2}=A$ and $\Delta(A)$ can be interpreted as the set of mixed strategies of player 2 in $B(p)$.

In this particular framework, a solution consists of a signalling strategy $\mu: K \rightarrow \Delta(S)$ for the informed player and a strategy $\chi: S \rightarrow \Delta(A)$ of the uninformed player. Furthermore,

$$
f_{1}(q)=\min _{\tau \in \Delta(A)} \sum_{k \in K} q^{k} U^{k}(\tau)=\min _{a \in A} \sum_{k \in K} q^{k} U^{k}(a)
$$

so that every feasible vector payoff is individually rational for player 1 . For the uninformed player, we have that

$$
g_{1}(q)=\max _{\tau \in \Delta(A)} \sum_{k \in K} q^{k} V^{k}(\tau)=\max _{a \in A} \sum_{k \in K} q^{k} V^{k}(a)
$$

and the individual rationality condition for this player reduces to

$$
\sum_{k} p_{s}^{k} V^{k}(\chi(s)) \geq \sum_{k \in K} p_{s}^{k} V^{k}(a) \text { for every } s \in S, a \in A
$$

where $p_{s}$ is player 2's posterior probability distribution on $K$ given $s$. It just says that given player 1's signal, player 2 chooses an action that maximizes his expected payoff. 
It thus appears that $(S, \mu, \chi)$ defines a solution for $B(p)$ if and only if $(\mu, \chi)$ is a Nash equilibrium of the following, noncooperative, sender-receiver game: player 1 is privately informed of his type $k$, then player 1 sends a signal $s \in S$ to player 2 and finally, player 2 makes a decision $a \in A$; player 1 gets $U^{k}(a)$ and player 2 gets $V^{k}(a)$. The uninformed player does not commit at all.

It is well-known (and easy to check) that every sender-receiver game has a nonrevealing equilibrium, namely, an equilibrium in which $\mu(s \mid k)$ does not depend on $k$. Hence, there always exists a nonrevealing solution in this case.

\subsection{2 "Known own" payoffs (private values)}

Being fully informed in $B(p)$, player 1 obviously knows his own payoff. In this section, we further assume that player 2's payoff does not depend on player 1's type and is just described by a mapping $V$, independent of $k \in K$. Let us define

$$
v^{*}=\min _{\sigma \in \Delta\left(A_{1}\right)} \max _{\tau \in \Delta\left(A_{2}\right)} V(\sigma, \tau)=\max _{\tau \in \Delta\left(A_{2}\right)} \min _{\sigma \in \Delta\left(A_{1}\right)} V(\sigma, \tau)
$$

In this simple framework, a joint decision $x \in \Delta(A)$ induces an individually rational payoff for the uninformed player in $B(p)$ if and only if $V(x) \geq v^{*}$.

A completely revealing solution in $B(p)$ is easily constructed. Let us take $S \equiv K, \mu(k \mid k)=1$ for every $k \in K$ (so that the posteriors correspond to the extreme points $\delta_{k}$ of $\left.\Delta(K)\right)$ and

$$
\chi(k)=x_{k} \in \arg \max \left\{U^{k}(x): x \in \Delta(A), V(x) \geq v^{*}\right\} \quad \forall k \in K
$$

It is immediate that any $(S, \mu, \chi)$ defined in this way satisfies incentive compatibility and individual rationality for player $2\left(V\left(x_{k}\right) \geq v^{*}\right.$ for every $\left.k\right)$. To show that it satisfies individual rationality for player 1 , recalling (4), let $\tilde{\sigma}(q)=\left(\sigma^{k}(q)\right)_{k \in K} \in \Delta\left(A_{1}\right)^{K}$ be a strategy of player 1 in the game $\Gamma_{1}(q)$ that guarantees him $f_{1}(q)$, let $\tau \in \Delta\left(A_{2}\right)$ be a strategy of player 2 that guarantees $\operatorname{him} v^{*}$ in the one-shot game $V$ and let $y_{k}(q)=\sigma^{k}(q) \otimes \tau$. By construction, $V\left(y_{k}(q)\right) \geq v^{*}$ for every $k \in K$. Hence, $U^{k}\left(x_{k}\right) \geq U^{k}\left(y_{k}(q)\right) \forall k \in K$ and $q \cdot u=\sum_{k} q^{k} U^{k}\left(x_{k}\right) \geq \sum_{k} q^{k} U^{k}\left(y_{k}(q)\right) \geq f_{1}(q)$. 


\subsection{Uniform punishment strategy}

Let us come back to the basic game $B(p)$ and introduce a property of player 1 's one-shot game payoffs $U^{k}(),. k \in K$. Recall that for every $k \in K, \underline{u}^{k}$ is defined as the individually rational level of player 1 of type $k$, when $k$ is complete information (see (8)).

Definition $8 \tau^{*} \in \Delta\left(A_{2}\right)$ is a uniform punishment strategy (UPS) of the uninformed player iff

$$
\forall k \in K \forall \sigma \in \Delta\left(A_{1}\right): U^{k}\left(\sigma, \tau^{*}\right) \leq \underline{u}^{k}
$$

The property means that the uninformed player can punish the informed one in the harshest way, namely, as if he knew the informed player's type, even without knowing it (see Forges and Salomon (2014)). UPS is satisfied in many games derived from economic applications: auctions, duopoly, contribution to a public good, etc. We will show that under UPS, there always exists a solution. ${ }^{9}$

As observed before, if a payoff vector $u=\left(u^{k}\right)_{k \in K}$ is individually rational for player 1 in $B(\cdot)$ (see definition 1), it is always true that $u^{k} \geq \underline{u}^{k} \forall k \in K$. If player 2 has a uniform strategy, the converse also holds, so that

Lemma 9 If the uninformed player has a uniform punishment strategy, a payoff vector $u=\left(u^{k}\right)_{k \in K}$ is individually rational for player 1 in $B(\cdot)$ iff $u^{k} \geq \underline{u}^{k} \forall k \in K$.

This characterization of player 1's individual rationality is familiar in principal-agent problems. Example 1 above shows that the existence of a uniform punishment strategy is crucial for the "if" part of the lemma.

Let $u=\left(u^{k}\right)_{k \in K}$ be player 1's interim expected payoff at a solution (namely, (1)): $u$ is individually rational for player 1 iff $U^{k}\left(x_{s}\right) \geq \underline{u}^{k}$ for every $k \in K$ and for every $s \in S$ such that $\mu(s \mid k)>0$, namely, if, at every signal $s$, the joint decision $x_{s}$ is posterior individually rational for player 1 . Individual rationality for player 1 can still be written equivalently by making

\footnotetext{
${ }^{9}$ UPS guarantees the existence of an equilibrium of the form described in lemma 6 but does not ensure that this equilibrium be Bayesian perfect. To see this, it suffices to modify example 1 by giving player 2 an additional action whose payoff is always negative for both players. Player 2 has then a strictly dominated uniform punishment strategy. Eliminating it recreates the non-existence problem.
} 
use of the mapping $f_{1}$ defined in (4), which, under UPS, takes the simple form

$$
f_{1}(q)=\sum_{k \in K} q^{k} \underline{u}^{k} \quad \forall q \in \Delta(K)
$$

Indeed, by (4) and (8), $f_{1}(q) \leq \sum_{k \in K} q^{k} \underline{u}^{k}$. Conversely, player 1 can guarantee himself $\sum_{k \in K} q^{k} \underline{u}^{k}$ by playing an optimal strategy in the (one-shot, zero-sum) game with payoffs $U^{k}(\cdot)$ when he is of type $k$, for every $k$.

We are now ready to state our main result (which holds without any restriction on player 2's payoffs):

Proposition 10 Let $B(p)$ be a two-person Bayesian game with a single informed player in which the uninformed player has a uniform punishment strategy. For every $p \in \Delta(K), B(p)$ has a feasible, posterior individually rational solution.

We show below that one way to establish proposition 10 is to see that it can be deduced from the existence of special Nash equilibria (namely, Aumann and Maschler (1968, 1995)'s joint plan equilibria) in the undiscounted infinitely repeated version $B_{\infty}(p)$ of the basic Bayesian game (see Simon et al. (1995)).

\section{A proof of proposition 10 relying on Simon et al. (1995)}

Let $B_{\infty}(p)$ be the following infinitely repeated game: at stage 0, player 1 's type $k$ is chosen once and for all according to $p$; at every stage $t=$ $1,2, \ldots$, player 1 and player 2 choose simultaneously an action in $A_{1}$ and $A_{2}$ respectively; the actions chosen at every stage are revealed to both players stage after stage; the stage payoffs are evaluated by $U^{k}($.$) and V^{k}($.$) and only$ known to player 1 . Payoffs in $B_{\infty}(p)$ are evaluated through (Banach) limits of means (see Hart (1985) and Forges (1992)).

In $B_{\infty}(p)$, player 1 can reveal his information through his actions. A simple signalling strategy $\mu$ for player 1 in $B_{\infty}(p)$ can thus be described as in section 2.1, with $S=\left(A_{1}\right)^{t}$ for some stage $t$. According to Aumann, Maschler and Stearns $(1968,1995)$ (see also Forges (1992)) a joint plan equilibrium in $B_{\infty}(p)$ consists of a signalling strategy $\mu$ for player 1 and for every signal $s$, of a nonrevealing equilibrium of $B_{\infty}\left(p_{s}\right)$ to be played from stage $t+1$ on, given the posterior probability distribution $p_{s}$ that is achieved through signalling. The nonrevealing equilibrium of $B_{\infty}\left(p_{s}\right)$ is described by a correlated 
strategy $^{10} x_{s} \equiv \chi(s) \in \Delta(A)$ which induces individually rational payoffs for both players in $B_{\infty}\left(p_{s}\right)$.

To formally define individual rationality in $B_{\infty}(q), q \in \Delta(K)$, let $\Gamma_{1, N R}(q)$ (resp., $\left.\Gamma_{2, N R}(q)\right)$ be the one-shot expected game based on player 1 (resp., 2)'s payoff and let $f_{N R}(q)$ (resp., $g_{N R}(q)$ ) be the value of $\Gamma_{1, N R}(q)$ (resp., $\left.\Gamma_{2, N R}(q)\right)$, namely,

$$
\begin{aligned}
f_{N R}(q) & =\min _{\tau \in \Delta\left(A_{2}\right)} \max _{\sigma \in \Delta\left(A_{1}\right)}\left(\sum_{k \in K} q^{k} U^{k}(\sigma, \tau)\right) \\
& =\max _{\sigma \in \Delta\left(A_{1}\right)} \min _{\tau \in \Delta\left(A_{2}\right)}\left(\sum_{k \in K} q^{k} U^{k}(\sigma, \tau)\right) \\
g_{N R}(q) & =\min _{\sigma \in \Delta\left(A_{1}\right)} \max _{\tau \in \Delta\left(A_{2}\right)}\left(\sum_{k \in K} q^{k} V^{k}(\sigma, \tau)\right) \\
& =\max _{\tau \in \Delta\left(A_{2}\right)} \min _{\sigma \in \Delta\left(A_{1}\right)}\left(\sum_{k \in K} q^{k} V^{k}(\sigma, \tau)\right)
\end{aligned}
$$

In both $f_{N R}$ and $g_{N R}$, it is understood that player 1 does not make use of his information, i.e., chooses his strategy independently of his type.

Let $p$ be any prior in the interior of $\Delta(K)$; following Blackwell (1956) and Aumann and Maschler $(1968,1995)$, a vector payoff $u=\left(u^{k}\right)_{k \in K}$ is individually rational for player 1 in $B_{\infty}(p)$ if and only if

$$
\forall q \in \Delta(K) \quad q \cdot u \geq f_{N R}(q)
$$

where $f_{N R}$ is defined by (11). In other words, the latter condition is necessary and sufficient for player 2 to have a strategy $\tau_{\infty}$ in $B_{\infty}(p)$ which guarantees that player 1's payoff in $B_{\infty}(p)$ does not exceed $u^{k}$ when his type is $k$, whatever the type $k$ is.

Similarly, a payoff $\bar{v}$ is individually rational for player 2 in $B_{\infty}(p)$ if and only if

$$
\bar{v} \geq v \operatorname{exg} g_{N R}(p)
$$

\footnotetext{
${ }^{10}$ In the context of $B_{\infty}(p)$, a correlated strategy in $\Delta(A)$ is interpreted as a limit frequency of moves.
} 
where $\operatorname{vex} g_{N R}$ is the convexification of $g$, i.e., the largest convex mapping that is below $g_{N R}$ over the simplex. In other words, (14) is necessary and sufficient for player 1 to have a (type dependent) strategy $\sigma_{\infty}$ in $\Gamma_{\infty}(p)$ which guarantees that player 2's payoff in $B_{\infty}(p)$ does not exceed $\bar{v}$.

Using the previous notions of individual rationality, we can give a fully precise definition: $(S, \mu, \chi)$ is a joint plan equilibrium in $B_{\infty}(p)$ iff

$(S, \mu, \chi)$ satisfies the incentive compatibility conditions i) and ii) of section 2.1 , with $U^{k}\left(x_{s}\right) \equiv U^{k}(\chi(s))$ for every $k, s$ such that $\mu(s \mid k)>0$.

Player 1's interim expected payoff $\left(u^{k}\right)_{k \in K}$, which can be defined as in (1), namely, by $u^{k}=U^{k}\left(x_{s}\right)$ for every $k, s$ such that $\mu(s \mid k)>0$, is individually rational in the sense of (13).

Player 2's conditional expected payoff given $s$, namely, $\bar{v}_{s}=\sum_{k} p_{s}^{k} V^{k}\left(x_{s}\right)$, where $p_{s}$ is the posterior probability distribution over $K$ at $s$ (computed from $p$ and $\mu$ ), is individually rational in $B_{\infty}\left(p_{s}\right)$, in the sense of (14).

According to Simon et al. (1995), for every $p$, there exists a joint plan equilibrium $(S, \mu, \chi)$ in the infinitely repeated game $B_{\infty}(p)$, which, by definition, is feasible (in particular, incentive compatible) in the one-shot game $B(p)$. To complete the proof of the proposition, we still have to check that $(S, \mu, \chi)$ is individually rational for both players in $B(p)$.

For player 2, the result is immediate. By lemma 4, individidual rationality for player 2 in $B(p)$ can be formulated in terms of the mapping $g_{1}$ (see (9)). By the definitions of $g_{1}$ and $g_{N R}, g_{1} \leq g_{N R}$ and $g_{1}$ is convex. Hence, for player 2 , individidual rationality in $B_{\infty}\left(p_{s}\right)$ implies individidual rationality in $B\left(p_{s}\right)$, at every $s$.

Let us show that, thanks to UPS, $(S, \mu, \chi)$ is also individually rational for player 1 in $B(p)$. To see this, let $\tau^{*}$ be a uniform strategy of player 2 ; by the definition of $f_{N R}$ (namely, (11)),

$$
f_{N R}(q) \leq \max _{\sigma \in \Delta\left(A_{1}\right)}\left(\sum_{k \in K} q^{k} U^{k}\left(\sigma, \tau^{*}\right)\right) \leq \sum_{k \in K} q^{k} \max _{\sigma \in \Delta\left(A_{1}\right)} U^{k}\left(\sigma, \tau^{*}\right) \leq \sum_{k \in K} q^{k} \underline{u}^{k} .
$$

This implies that under UPS, $\operatorname{cav}_{N R}(q) \leq \sum_{k \in K} q^{k} \underline{u}^{k}$, where $\operatorname{cav} f_{N R}$ is the concavification of $f_{N R}$, i.e., the smallest concave mapping that is above 
$f_{N R}$ over the simplex. On the other hand, recalling that $\delta_{k}$ denotes the $k$ th extreme point of $\Delta(K)$,

$$
\begin{aligned}
\operatorname{cavf}_{N R}(q) & =\operatorname{cavf}_{N R}\left(\sum_{k \in K} q^{k} \delta_{k}\right) \\
& \geq \sum_{k \in K} q^{k} \operatorname{cavf} f_{N R}\left(\delta_{k}\right)=\sum_{k \in K} q^{k} f_{N R}\left(\delta_{k}\right)=\sum_{k \in K} q^{k} \underline{u}^{k} .
\end{aligned}
$$

Hence, under UPS,

$$
\operatorname{cavf}_{N R}(q)=\sum_{k \in K} q^{k} \underline{u}^{k} \quad \forall q \in \Delta(K)
$$

and, using (13), $u=\left(u^{k}\right)_{k \in K}$ is individually rational for player 1 in $B_{\infty}(p)$ iff

$$
u^{k} \geq \underline{u}^{k} \quad \forall k \in K .
$$

From lemma 9, under UPS, individual rationality for player 1 in the one-shot game $B(p)$ reduces to the same condition.

The previous proof shows that when UPS holds, $c a v f_{N R}$ is linear. ${ }^{11}$ The latter property greatly simplifies the study of infinitely repeated games with lack of information on one side since it allows to dispense with Blackwell's approachability theorem in the definition of individual rationality for the informed player. However, another look at example 1 shows that the linearity of $\operatorname{cavf}_{N R}$ is weaker than UPS and does not guarantee the existence of a solution in $B(p)$.

Example 1 (continued): Recall that, in this example,

$$
f_{1}(q)=\min \{q+2(1-q), 2 q+(1-q)\}
$$

so that a vector payoff $\left(u^{1}, u^{2}\right)$ is individually rational for player 1 in $B(\cdot)$ iff $u^{1}+u^{2} \geq 3$. Given player 2's payoffs, there is no cooperative solution in $B(p), 0<p<1$. One computes that

$$
\begin{aligned}
f_{N R}(p) & =1-p \text { if } p \leq \frac{1}{3} \\
& =3 p(1-p) \text { if } \frac{1}{3} \leq p \leq \frac{2}{3} \\
& =p \text { if } p \geq \frac{2}{3}
\end{aligned}
$$

\footnotetext{
${ }^{11}$ More precisely, recalling the definition of the concave mapping $f_{1}$ (i.e., (4)) and lemma 9, under UPS, $\operatorname{cav}_{N R}(q)=f_{1}(q)=\sum_{k \in K} q^{k} \underline{u}^{k}$.
} 
Hence, $\operatorname{cav} f_{N R}(p)=1$ for every $p$. In particular, $c a v f_{N R}$ is linear, even if UPS is not satisfied. For every $p, B_{\infty}(p)$ has a completely revealing equilibrium achieving the vector payoff $(1,1)$ for player 1 . This example illustrates that when UPS does not hold, individual rationality for player 1 is weaker in $B_{\infty}(\cdot)$ than in $B(\cdot)$.

In the next example, UPS is verified and a cooperative solution exists. However, for some values of the prior probability distribution over states, there does not exist any nonrevealing solution and there does not exist any completely revealing solution either. In particular, the revelation principle does not hold in our framework. In the partially revealing constructed solution, the informed player of type $k$ just gets his individually rational level $\underline{u}^{k}$.

Example 2: Let $K=\{1,2\}, A_{1}=\{T, B\}, A_{2}=\{F L, L, C, R, F R\}$. Player 1's payoff matrices are:

$$
\begin{array}{ccccccc}
U^{1}(\cdot)= & & F L & L & C & R & F R \\
& T & 4 & 3 & 4 & 3 & 4 \\
B & 0 & 3 & 0 & 3 & 8 \\
U^{2}(\cdot)= & & F L & L & C & R & F R \\
& & 8 & 3 & 0 & 3 & 0 \\
B & 4 & 3 & 4 & 3 & 4
\end{array}
$$

We check that $\underline{u}^{1}=\underline{u}^{2}=3$ and that $L$ and $R$ are uniform punishment strategies of player 2. Player 2's payoffs are exactly as in Forges (1990); in particular, they depend on player 1's type but they do not depend on player 1's action:

$$
\begin{array}{rcccccc}
V^{1}(\cdot)= & & F L & L & C & R & F R \\
& & 10 & 9 & 7 & 4 & 0 \\
B & 10 & 9 & 7 & 4 & 0 \\
V^{2}(\cdot)= & & F L & L & C & R & F R \\
& T & 0 & 4 & 7 & 9 & 10 \\
B & 0 & 4 & 7 & 9 & 10
\end{array}
$$

Let us denote as $p \in[0,1]$ the probability that $k=1$. It is immediate that $F R$ is optimal for $0 \leq p \leq \frac{1}{5}, R$ is optimal for $\frac{1}{5} \leq p \leq \frac{2}{5}, C$ is optimal for $\frac{2}{5} \leq p \leq \frac{3}{5}, L$ is optimal for $\frac{3}{5} \leq p \leq \frac{4}{5}, F L$ is optimal for $\frac{4}{5} \leq p \leq 1$. 
Let $p=\frac{1}{2}$. Let us first show that there is no nonrevealing solution. A nonrevealing solution consists of $x \in \Delta(A)$ such that $U^{1}(x) \geq 3, U^{2}(x) \geq 3$ and $\frac{1}{2} V^{1}(x)+\frac{1}{2} V^{2}(x) \geq g_{1}\left(\frac{1}{2}\right)=7$. The latter condition implies that player 2 must take the action $C$, namely that

$$
x=\left(\begin{array}{ccccc}
0 & 0 & \alpha & 0 & 0 \\
0 & 0 & 1-\alpha & 0 & 0
\end{array}\right) \quad \text { for some } 0 \leq \alpha \leq 1
$$

$U^{1}(x) \geq 3 \Rightarrow \alpha \geq \frac{3}{4}$ and $U^{2}(x) \geq 3 \Rightarrow \alpha \leq \frac{1}{4}$ so that there is indeed no nonrevealing solution.

Let us show next that there is no completely revealing solution at $p=\frac{1}{2}$. A completely revealing solution is described by $x_{k} \in \Delta(A), k=1,2, x_{k}$ being interpreted as the joint decision when player 1 reports type $k$. Individual rationality for player 2 means that $V^{1}\left(x_{1}\right) \geq g_{1}(1)$, i.e., player 2 chooses $F L$ when he gets signal 1 and similarly, $V^{2}\left(x_{2}\right) \geq g_{1}(0)$, i.e., player 2 chooses $F R$ when he gets signal 2 .

$$
\begin{array}{lll}
x_{1}=\left(\begin{array}{ccccc}
\alpha & 0 & 0 & 0 & 0 \\
1-\alpha & 0 & 0 & 0 & 0
\end{array}\right) & \text { for some } 0 \leq \alpha \leq 1 \\
x_{2}=\left(\begin{array}{ccccc}
0 & 0 & 0 & 0 & 1-\beta \\
0 & 0 & 0 & 0 & \beta
\end{array}\right) & \text { for some } 0 \leq \beta \leq 1
\end{array}
$$

Individual rationality for player 1 means that $U^{k}\left(x_{k}\right) \geq 3, k=1,2$, i.e., $U^{1}\left(x_{1}\right)=4 \alpha$ with $\alpha \geq \frac{3}{4}$ and $U^{2}\left(x_{2}\right)=4 \beta$ with $\beta \geq \frac{3}{4}$. Incentive compatibility for player 1 means that $U^{1}\left(x_{1}\right) \geq U^{1}\left(x_{2}\right)$ and $U^{2}\left(x_{2}\right) \geq U^{2}\left(x_{1}\right) \Leftrightarrow 4 \alpha \geq$ $4(1-\beta)+8 \beta$ and $4 \beta \geq 8 \alpha+4(1-\alpha) \Leftrightarrow \alpha \geq 1+\beta$ and $\beta \geq 1+\alpha$, which is of course impossible.

Let us show that there exists a partially revealing solution at $p=\frac{1}{2}$. Let player 1 send signals $r$ and $s$ so as to reach the posteriors $p_{r}=\frac{1}{4}$ and $p_{s}=\frac{3}{4}$. In order to satisfy player 2's individual rationality condition, we must have that $x_{r}$ gives probability 1 to $R$ and that $x_{s}$ gives probability 1 to $L$. Incentive compatibility is trivially satisfied since player 1 gets 3 no matter what. The payoffs at this partially revealing solution are $(3,3)$ for player 1 (i.e., just his individual rationality level) and $\frac{31}{4}$ for player 2 .

To complete the analysis,

$$
\begin{aligned}
f_{N R}(p) & =4 \max \{p, 1-p\} \quad \text { if } \frac{1}{4} \leq p \leq \frac{3}{4} \\
& =3 \text { otherwise }
\end{aligned}
$$


so that $\operatorname{cav}_{N R}(p)=3$ for every $p$.

As another reference, the one-shot (noncooperative) Bayesian game has a Nash equilibrium in which player 1 chooses $T$ when $k=1$ and $B$ when $k=2$, while player 2 chooses $C$, which gives the payoffs $(4,4)$ to player 1 and 7 to player 2 . These payoffs can be achieved by a centralized mechanism à la Myerson (1991), i.e., they are in $\mathcal{M}\left[B\left(\frac{1}{2}\right)\right]$ (see section 2.4) but are not in $\mathcal{C}\left[B\left(\frac{1}{2}\right)\right]$ because player 1 makes use of his private information without sharing it with player 2 .

\section{Concluding remarks}

\subsection{Further insights when a single player is informed}

Let us first summarize some properties that do not rely on UPS. In section 2, we have defined two sets of cooperative payoffs in the Bayesian game $B(p)$ : the set $\mathcal{C}[B(p)]$ of unmediated, posterior individually rational payoffs (definition 5) and the set $\mathcal{M}[B(p)]$ of mediated, interim individually rational payoffs (definition 7). We have seen that $\mathcal{C}[B(p)] \subseteq \mathcal{M}[B(p)]$ and that this inclusion may be strict. Recalling the proof of proposition 10, let $\mathcal{N}_{J P}\left[B_{\infty}(p)\right] \subseteq \mathbb{R}^{K} \times \mathbb{R}$ be the set of all interim expected payoff that can be achieved in a joint plan equilibrium of $B_{\infty}(p)$. Simon et al. (1995)'s result can be stated as $\mathcal{N}_{J P}\left[B_{\infty}(p)\right] \neq \emptyset$.

When UPS is satisfied, the findings of section 3.3 can be rephrased as follows:

Corollary 11 Let $B(p)$ be a two-person Bayesian game with a single informed player in which the uninformed player has a uniform punishment strategy. $\mathcal{N}_{J P}\left[B_{\infty}(p)\right]=\mathcal{C}[B(p)] \subseteq \mathcal{M}[B(p)]$. Furthermore, $\mathcal{C}[B(p)]$ is nonempty and may be strictly included in $\mathcal{M}[B(p)]$.

The strict inclusion of $\mathcal{C}[B(p)]$ in $\mathcal{M}[B(p)]$ under UPS is illustrated on example 2. Viewed as a result on $\mathcal{N}_{J P}\left[B_{\infty}(p)\right]$, the previous corollary can be interpreted as an extension of the folk theorem to games with incomplete information, since it makes a precise connection between a set of (noncooperative) equilibrium payoffs of the infinitely repeated game and sets of feasible individually rational payoffs of the one-shot game. For the set $\mathcal{C}[B(p)]$ of unmediated feasible, posterior individually rational payoffs, we have a full folk theorem, in the sense that $\mathcal{N}_{J P}\left[B_{\infty}(p)\right]$ coincides with $\mathcal{C}[B(p)]$. For the 
set $\mathcal{M}[B(p)]$ of mediated feasible, interim individually rational payoffs, we just have a partial folk theorem.

We have seen that when UPS does not hold, $\mathcal{C}[B(p)]$ can be empty, while $\mathcal{N}_{J P}\left[B_{\infty}(p)\right]$ is still nonempty. The partial folk theorem property $\mathcal{N}_{J P}\left[B_{\infty}(p)\right] \subseteq$ $\mathcal{M}[B(p)]$ can also fail when UPS is not satisfied. This is illustrated in section 3.3: in example 1 (continued), $B_{\infty}(p)$ has a completely revealing equilibrium which is obviously individually rational for player 1 in $B_{\infty}(p)$ (namely, satisfies (13)) but is not interim individually rational for player 1 in $B(p)$, in spite of the fact that player 2's approachability strategy is straightforward, cav $f_{N R}$ being linear.

\subsection{Solutions when both players are privately informed}

Let us extend the basic Bayesian game so that both player 1 and player 2 have private information. Let $K$ and $L$ be finite sets. At a virtual initial stage of the game, player 1's type $k$ is chosen in $K$ according to $p$ and, independently, player 2's type $\ell$ is chosen in $L$ according to $q$. Only player 1 (resp., 2) is informed of $k$ (resp., $\ell$ ). Unless specified otherwise, we assume that $p^{k}>0$ and that $q^{\ell}>0$ for every $k \in K, \ell \in L$.

Player 1 and player 2 choose simultaneously an action in finite sets $A_{1}$ and $A_{2}$ respectively. If the pair of actions $a \in A=A_{1} \times A_{2}$ is chosen, they get the respective payoffs $U^{k}(a)$ and $V^{\ell}(a)$. Each player thus knows his own payoff (according to another terminology, "values are private"). We refer to this game as $B(p, q)$.

We will also assume that UPS holds for both players. Given the private values assumption, we can still define player 1's type $k$ individually rational level by $\underline{u}^{k}$, exactly as in (8), for every $k \in K$, and we can define player 2 's type $\ell$ individually rational level in the same way, by $\underline{v}^{\ell}$, for every $\ell \in L$.

Let $S$ and $T$ be sets of signals and let $\chi: S \times T \rightarrow \Delta(A)$. As in section 2.3 , we can introduce a noncooperative game $G(p, q, S, T, \chi)$ to be played as follows:

- Player 1's type $k$ is chosen in $K$ according to $p$, player 2's type $\ell$ is chosen in $L$ according to $q$, only player 1 (resp., 2) is informed of $k$ (resp., $\ell$ ).

- Player 1 sends a signal $s \in S$ to player 2, and simultaneously, player 2 sends a signal $t \in T$ to player 1 .

- The correlated strategy $\chi(s, t)=x_{s t} \in \Delta(A)$ is proposed to both players. 
- The players simultaneously accept or reject the correlated strategy $x_{s t}$.

- If both players accept $x_{s t}$, player 1 gets $U^{k}\left(x_{s t}\right)$ and player 2 gets $V^{\ell}\left(x_{s t}\right)$.

- If at least one of the players rejects $x_{s t}$, player 1 chooses $a_{1}$, player 2 chooses $a_{2}$, player 1 gets $U^{k}\left(a_{1}, a_{2}\right)$ and player 2 gets $V^{\ell}\left(a_{1}, a_{2}\right)$.

Let $\mu: K \rightarrow \Delta(S)$ be a signalling strategy for player 1 and let $\nu$ : $L \rightarrow \Delta(T)$ be a signalling strategy for player 2 . By proceeding as in section 2.3 , we say that $(S, T, \mu, \nu, \chi)$ defines a solution for $B(p, q)$ if and only if $G(p, q, S, T, \chi)$ has an equilibrium in which player 1 sends his signal according to $\mu$, player 2 sends his signal according to $\nu$ and both players always accept the correlated strategy that is proposed to them.

Let us further say that $(S, T, \mu, \nu, \chi)$ defines a completely revealing (cooperative) solution if $S=K, T=L, \mu$ (resp., $\nu$ ) is the identity over $K$ (resp., $L)$. A completely revealing solution is thus just described by $x_{k \ell} \in \Delta(A)$ for every $(k, \ell) \in K \times L$. It can be checked that $\left(x_{k \ell}\right)_{(k, \ell) \in K \times L}$ defines a completely revealing solution if and only if the following inequalities hold

$$
\begin{array}{ll}
\sum_{\ell \in L} q^{\ell} U^{k}\left(x_{k \ell}\right) \geq \sum_{\ell \in L} q^{\ell} \max \left\{U^{k}\left(x_{k^{\prime} \ell}\right), \underline{u}^{k}\right\} & \forall k, k^{\prime} \in K \\
\sum_{k \in K} p^{k} V^{\ell}\left(x_{k \ell}\right) \geq \sum_{k \in K} p^{k} \max \left\{V^{\ell}\left(x_{k \ell^{\prime}}\right), \underline{v}^{\ell}\right\} & \forall \ell, \ell^{\prime} \in L
\end{array}
$$

Let $\mathcal{C}[B(p, q)] \subseteq \mathbb{R}^{K} \times \mathbb{R}^{L}$ be the set of all interim payoffs that can be achieved at a solution of $B(p, q)$. The next lemma states that all payoffs $(u, v)=\left(\left(u^{k}\right)_{k \in K},\left(v^{\ell}\right)_{\ell \in L}\right)$ in $\mathcal{C}[B(p, q)]$ are of the form

$$
u^{k}=\sum_{\ell \in L} q^{\ell} U^{k}\left(x_{k \ell}\right), v^{\ell}=\sum_{k \in K} p^{k} V^{\ell}\left(x_{k \ell}\right)
$$

for some $\left(x_{k \ell}\right)_{(k, \ell) \in K \times L}$ satisfying inequalities (15).

Lemma 12 Let $B(p, q)$ be a two-person Bayesian game with private values in which both players have a uniform punishment strategy. Every solution for $B(p, q)$ is payoff equivalent to a completely revealing solution.

Proof: Let $(S, T, \mu, \nu, \chi)$ be a solution for $B(p, q)$. Let us consider the variant of $G(p, q, S, T, \chi)$ in which both players are invited to reveal their types to a mediator who selects $x \in \Delta(A)$ according to the probability distribution

$$
\rho(x \mid k, \ell)=\sum_{s \in S, t \in T} \mu(s \mid k) \nu(t \mid \ell) I\left(x=x_{s t}\right)
$$


and asks them whether they accept $x$ or not; if they both accept, $x$ is implemented; otherwise, they choose their actions themselves. By proceeding in the same way as to establish the revelation principle, it is a Nash equilibrium for both players to reveal their type and to accept the outcome proposed by the mediator. ${ }^{12}$ We thus have, for player 1 ,

$$
\sum_{\ell \in L} q^{\ell} \sum_{x} \rho(x \mid k, \ell) U^{k}(x) \geq \sum_{\ell \in L} q^{\ell} \sum_{x} \rho\left(x \mid k^{\prime}, \ell\right) \max \left\{U^{k}(x), \underline{u}^{k}\right\} \quad \forall k, k^{\prime} \in K
$$

Let us set

$$
x_{k \ell}=\sum_{x} \rho(x \mid k, \ell) x \equiv E_{\rho}(\widetilde{x} \mid k, \ell)
$$

Then, recalling that $U^{k}$ is linear, the left hand side of (16) is $\sum_{\ell \in L} q^{\ell} U^{k}\left(x_{k \ell}\right)$. By using that $\max$ is convex, and then again that $U^{k}$ is linear, the right hand side is larger than $\sum_{\ell \in L} q^{\ell} \max \left\{U^{k}\left(\sum_{x} \rho\left(x \mid k^{\prime}, \ell\right) x\right), \underline{u}^{k}\right\}$, hence (16) implies (15). One can proceed similarly for player 2 .

Equipped with the previous simple characterization of $\mathcal{C}[B(p, q)]$, we are going to check whether an analog of corollary 11 holds in the current model. Koren (1992) already proposed a characterization like lemma 12 for the undiscounted infinitely repeated version of $B(p, q)$, which we denote as $B_{\infty}(p, q)$ and which can be defined in the same way as $B_{\infty}(p)$. Assuming private values but not UPS, Koren (1992) showed that every Nash equilibrium of $B_{\infty}(p, q)$ is payoff equivalent to a completely revealing Nash equilibrium, so that it can in particular be achieved as a joint plan equilibrium.

Let $\mathcal{N}\left[B_{\infty}(p, q)\right]$ be the set of all interim expected Nash equilibrium payoffs of $B_{\infty}(p, q)$. Defining $\mathcal{N}_{J P}\left[B_{\infty}(p, q)\right]$ in the same way as $\mathcal{N}_{J P}\left[B_{\infty}(p)\right]$ in section 3.3, we have here $\mathcal{N}\left[B_{\infty}(p, q)\right]=\mathcal{N}_{J P}\left[B_{\infty}(p, q)\right]$. Forges and Salomon (2014) further show that, when UPS holds, $\mathcal{N}\left[B_{\infty}(p, q)\right]$ is characterized by inequalities (15), i.e., that $\mathcal{N}\left[B_{\infty}(p, q)\right]=\mathcal{C}[B(p, q)] .{ }^{13}$

The set $\mathcal{M}[B(p, q)]$ of all mediated interim indivually rational payoffs when both players are informed can be defined as in section 2.4. A partial analog of corollary 11 is as follows:

\footnotetext{
${ }^{12}$ Since values are private and utility functions are linear, a player only cares about his own type and the expected distribution over actions.

${ }^{13}$ Koren (1992)'s characterization is more complex in that it makes use of Blackwell's approachability, i.e., individual rationality is defined as in (13).
} 
Proposition 13 Let $B(p, q)$ be a two-person Bayesian game with private values in which both players have a uniform punishment strategy. $\mathcal{N}\left[B_{\infty}(p, q)\right]=$ $\mathcal{C}[B(p, q)] \subseteq \mathcal{M}[B(p, q)]$.

As corollary 11, the previous result can be interpreted as a folk theorem. However, there is an important difference with the case of a single informed player: $\mathcal{N}\left[B_{\infty}(p, q)\right]$ - and thus also $\mathcal{C}[B(p, q)]$ - can be empty. Koren (1992) provides a counter-example, which turns out to satisfy UPS (see Forges and Salomon (2014) for further illustration).

Observe that the model in proposition 13 is both more general and more restrictive than the one of corollary 11. In proposition 13, there is lack of information on both sides but values are private, which was not necessarily true (for player 2) above, except in section 3.2.2. The next example illustrates that even if player 1 is the only one to have private information and player 2 knows his own payoff (as in section 3.2.2) the partial folk theorem $\mathcal{N}\left[B_{\infty}(p, q)\right] \subseteq \mathcal{M}[B(p, q)]$ is not true without UPS. ${ }^{14}$

\section{Example 3}

Let $\left|A_{1}\right|=\left|A_{2}\right|=2$ and the utility functions be described by

$$
\begin{gathered}
U^{1}(\cdot)=\left(\begin{array}{ll}
1 & 0 \\
0 & 0
\end{array}\right) \quad U^{2}(\cdot)=\left(\begin{array}{ll}
0 & 0 \\
0 & 1
\end{array}\right) \\
V(\cdot)=\left(\begin{array}{ll}
0 & 2 \\
0 & 0
\end{array}\right)
\end{gathered}
$$

The assumption of uniform punishments is clearly not satisfied: player 2 must play right in order to hold player 1 of type 1 at his value level $\underline{u}_{1}=0$ and must play left to hold him at $\underline{u}_{2}=0$. Recalling (11), the value of player 1 's expected one-shot game is

$$
f_{N R}(p)=\operatorname{val}_{1}\left(\begin{array}{cc}
p & 0 \\
0 & 1-p
\end{array}\right)=p(1-p)
$$

\footnotetext{
${ }^{14}$ The undiscounted infinitely repeated version of the model of section 3.2.2. (namely, lack of information on one side and private values) was studied by Shalev (1994). He showed that all Nash equilibria are payoff equivalent to completely revealing ones (the result that was extended by Koren (1992)) and that Nash equilibria always exist (the reasoning in section 3.2.2 is similar to Shalev (1994)'s one).
} 
The probability distribution

$$
x_{1}=x_{2}=x=\left(\begin{array}{cc}
\frac{1}{4} & \frac{1}{2} \\
0 & \frac{1}{4}
\end{array}\right) \in \Delta(A)
$$

defines a nonrevealing equilibrium of $B_{\infty}(q)$, for every $p \in(0,1)$. Indeed, player 1's associated vector payoff $\left(u^{1}, u^{2}\right)=\left(\frac{1}{4}, \frac{1}{4}\right)$ satisfies the individual rationality condition (13) and player 2's payoff $v=1$ is individually rational since the value of player 2's game is $v^{*}=0$. Hence $\left(\left(\frac{1}{4}, \frac{1}{4}\right), 1\right) \in \mathcal{N}\left[B_{\infty}(p)\right]$ for every $p \in(0,1)$.

However, $\left(\left(\frac{1}{4}, \frac{1}{4}\right), 1\right) \notin \mathcal{M}[B(p)]$ because $\left(\frac{1}{4}, \frac{1}{4}\right)$ is not interim individually rational. Let $\tau=(\beta, 1-\beta)$; $\max _{a_{1}} U^{1}\left(a_{1}, \tau\right)=\beta \leq \frac{1}{4}$ is incompatible with $\max _{a_{1}} U^{2}\left(a_{1}, \tau\right)=1-\beta \leq \frac{1}{4}$.

The example also allows us to recall that conditions (15) are not meaningful when UPS does not hold. The probability distribution

$$
x_{1}=x_{2}=x=\left(\begin{array}{ll}
0 & 1 \\
0 & 0
\end{array}\right)
$$

satisfies (15) but the vector payoff of player 1 is $(0,0)$ and is not individually rational for player 1 (not only in the interim sense but even in $B_{\infty}(p)$ ).

\section{References}

[1] Aumann, R. J. and M. Maschler (1995), Repeated Games of Incomplete Information, Cambridge: M.I.T. Press.

[2] Aumann, R. J., M. Maschler and R. Stearns (1968), "Repeated games with incomplete information: an approach to the nonzero sum case", Reports to the U.S. Arms Control and Disarmament Agency, ST-143, Chapter IV, 117-216.

[3] Blackwell, D. (1956), "An analog of the minimax theorem for vector payoffs", Pacific Journal of Mathematics 10, 1-8.

[4] Celik, G. and M. Peters (2011), "Equilibrium rejection of a mechanism", Games and Economic Behavior 73, 375-387.

[5] Forges, F. (1990), "Equilibria with communication in a job market example", Quarterly Journal of Economics 105, 375-398. 
[6] Forges, F. (1992), "Repeated games of incomplete information : nonzero-sum", in: R. Aumann and S. Hart, Handbook of Game Theory with Economic Applications, Elsevier Science Publishers, NorthHolland, chapter 6, 155-177.

[7] Forges, F. (2013), "A folk theorem for Bayesian games with commitment", Games and Economic Behavior 78, 64-71.

[8] Forges, F. and A. Salomon (2014), "Bayesian repeated games and reputation", mimeo, Université Paris-Dauphine.

[9] Hart S. (1985), "Nonzero-sum two-person repeated games with incomplete information", Mathematics of Operations Research 10, 117-153.

[10] Kalai, A. and E. Kalai (2013), "Cooperation in two-person games, revisited", Quarterly Journal of Economics, 128, 917-966.

[11] Kalai, A., E. Kalai, E. Lehrer and D. Samet (2010), "A commitment folk theorem", Games and Economic Behavior 69, 127-137.

[12] Koren, G. (1992), "Two-person repeated games where players know their own payoffs", mimeo, Courant Institute of Mathematical Sciences, New York University.

[13] Myerson, R. (1991), Game theory: analysis of conflict, Harvard University Press.

[14] Peters, M. and B. Szentes (2012), "Definable and contractible contracts", Econometrica 80, 363-411.

[15] Renault, J. (2000), "On two-player repeated games with lack of information on one side and state-dependent signalling", Mathematics of Operations Research 25, 552-572.

[16] Shalev, J. (1994), "Nonzero-sum two-person repeated games with incomplete information and known-own payoffs", Games and Economic Behavior 7, 246-259.

[17] Simon R. (2002), "Separation of joint plan equilibrium payoffs from the min-max functions", Games and Economic Behavior 41, 79-102. 
[18] Simon R., S. Spież and H. Toruńczyk, (1995), "The existence of equilibria in certain games, separation for families of convex functions and a theorem of Borsuk-Ulam type", Israel Journal of Mathematics 92, 1-21.

[19] Simon R., S. Spież and H. Toruńczyk, (2008), "Equilibria in a class of games and topological results implying their existence", RACSAM 102, 161-179.

[20] Sorin, S. (1983), "Some results on the existence of Nash equilibria for non-zero sum games with incomplete information", International Journal of Game Theory 12, 193-205.

[21] Sorin, S. (2002), "A first course on zero-sum repeated games", Mathématiques et Applications 37, Springer.

[22] Tennenholtz, M. (2004), "Program equilibrium", Games and Economic Behavior 49, 363-373. 